\title{
DELIVERY OF NEBULISED DRUGS USING ENDOTRACHEAL TUBE
}

\author{
Agata Penconek, Marcin Odziomek, Agata Niedzielska and Arkadiusz Moskal \\ Warsaw University of Technology, Faculty of Chemical and Process Engineering, Waryńskiego 1, \\ 00-645 Warszawa, Poland
}

\begin{abstract}
The purpose of the studies was to estimate efficiency of delivering nebulised drugs into the lower respiratory tract through endotracheal tubes (ET tubes) which are commonly used in the treatment of uncooperative patients. Water solution of Disodium Cromoglycate (DSCG) was nebulised with a constant air flow $(25 \mathrm{l} / \mathrm{min})$. Experimental studies were done for eight ET tubes with varying sizes (internal diameter, length) and made of two different materials. Size distribution of aerosol leaving ET tubes was determined with the use of aerosol spectrometer. Fine Particle Fraction $(F P F)$ and Mass Median Aerodynamic Diameter (MMAD) were calculated for the aerosol leaving each tube. Additionally, mass of the Disodium Cromoglycate deposited into each endotracheal tube was determined. ET tubes can significantly influence the parameters of delivered aerosol depending on their diameter. FPF of aerosol delivered in to the respiratory tract is lower if small endotracheal tubes are used. However, MMAD and FPF for large endotracheal tubes are almost identical with $M M A D$ and $F P F$ from nebuliser. The results indicate that a substantial fraction of large droplets is eliminated from the aerosol stream in long endotracheal tubes $(270 \mathrm{~mm})$. In this case the mass of drug delivered through ET tubes is reduced but the content of small droplets increases (high value of $F P F)$.
\end{abstract}

Keywords: aerosol therapy, drug delivery, size distribution, deposition, endotracheal tube

\section{INTRODUCTION}

Aerosol therapy is an effective form of treatment of respiratory tract diseases. This technique allows a delivery of medications into a deep part of human lungs, avoiding side effects and minimising drugs dose (Sosnowski, 2010). Dry powder inhalers (DPI) and metered dose inhalers (MDI), as well as nebulisers are widely used in aerosol therapy, but they require coordination.

Aerosol therapy is used in treatment of asthma, cystic fibrosis, pulmonary fibrosis, bronchitis etc. It is also used to treat diseases of patients in comma, and then the use of endotracheal tubes (ET tubes) and nebulisers is required. Endotracheal tubes are used for invasive ventilation if patient is unable to breathe independently or to keep a patient's trachea open.

A wide range of ET tubes diameters and lengths allows to use endotracheal tubes for therapy of adults as well as children or even newborns. However, the efficiency of drug delivery into lungs through ET tubes may be different for different patients since several factors influence it. (Dhand, 2000). The main factors to be taken into consideration if endotracheal tubes are used to drug delivery are the type of nebuliser, the kind of medications, endotracheal tube diameter and its electrostatic charge, breathing conditions and patient's age. 
In vivo estimation of particles' deposition into respiratory tract with a use of ET tubes is a very difficult, ethically complicated and cost-intensive process. Nevertheless, a few studies on aerosol delivery to children, ventilated newborns and adults can be found in the literature (Arhens et al., 1986; Grigg et al., 1992; MacIntyre et al., 1985; Schueepp et al., 2009; Watterberg et al., 1991). Researchers found that $22.3-59.7 \%$ of inhaled medications are excreted in the urine and $0.064-48.6 \%$ are deposited into lungs, depending on drugs, equipment and methods. Computational studies (Mazela et al., 2011; Żywczyk et al., 2010) indicated that efficiency of medications' penetration into the lungs through ET tubes strongly depends on the size of particles. It may reach nearly $100 \%$ for small particles, but for large particles $\left(d_{p}>7 \mu \mathrm{m}\right)$ it equals almost $0 \%$. However, neither in vivo studies, nor in silico studies showed medication droplet diameter distribution at the end of ET tubes whereas the size distribution seems to be as important as drug dose.

The goal of this study was to determine the effectiveness of aerosol delivery through endotracheal tubes with the inner diameter between $2-6 \mathrm{~mm}$ and length of $160-270 \mathrm{~mm}$. Drug dose and medication droplet diameter distribution were taken into account. To the best of our knowledge this is the first study that combines these two factors.

\section{MATERIALS AND METHODS}

Micronized disodium cromoglycate - DSCG (GlaxoSmithKline Pharmaceuticals SA) was selected as the test material. It is safe and may be easily identified by spectrophotometry at wavelength $\lambda=340 \mathrm{~nm}$. An aqueous solution of DSCG was prepared with the use of reverse osmosis purified water (Puricom).

Water solution of DSCG was nebulised with the use of a Sidestream nebuliser connected to a compressor (MEDBRYT, model Monsun MP1). The airflow rate was established on $20 \mathrm{l} / \mathrm{min}$. An additional source of dry air $(5 \mathrm{l} / \mathrm{min})$ was installed between the nebuliser and endotracheal tubes.

We compared aerosol delivery through endotracheal tube of internal diameter: $2,2.5,3,4,4.5,5,5.5$, $6 \mathrm{~mm}$ and three different lengths (Table 1).

Table 1. Sizes of endotracheal tubes

\begin{tabular}{|c|c|c|c|}
\hline \multicolumn{2}{|c|}{ ET tube } & $\begin{array}{c}\text { Internal diameter } \\
{[\mathrm{mm}]}\end{array}$ & $\begin{array}{c}\text { Length } \\
{[\mathrm{mm}]}\end{array}$ \\
\hline 520.20 & 70516.20 & 2.0 & 165 \\
\hline 520.25 & 70516.25 & 2.5 & 165 \\
\hline 520.30 & 70516.30 & 3.0 & 165 \\
\hline 520.40 & 70516.40 & 4.0 & 230 \\
\hline 520.45 & 70516.45 & 4.5 & 230 \\
\hline 520.50 & 70516.50 & 5.0 & 230 \\
\hline 520.55 & 70516.55 & 5.5 & 270 \\
\hline 520.60 & 70516.60 & 6.0 & 270 \\
\hline
\end{tabular}

Two types of ET tubes (520.xx and 70516.xx) provided by Vygon Corporation were chosen. Endotracheal tube 520.xx is made of extra-soft polivinylochloride (PVC), whereas endotracheal tube 70516.xx is made of PVC and silicon. The endotracheal tube is presented in Fig. 1. 


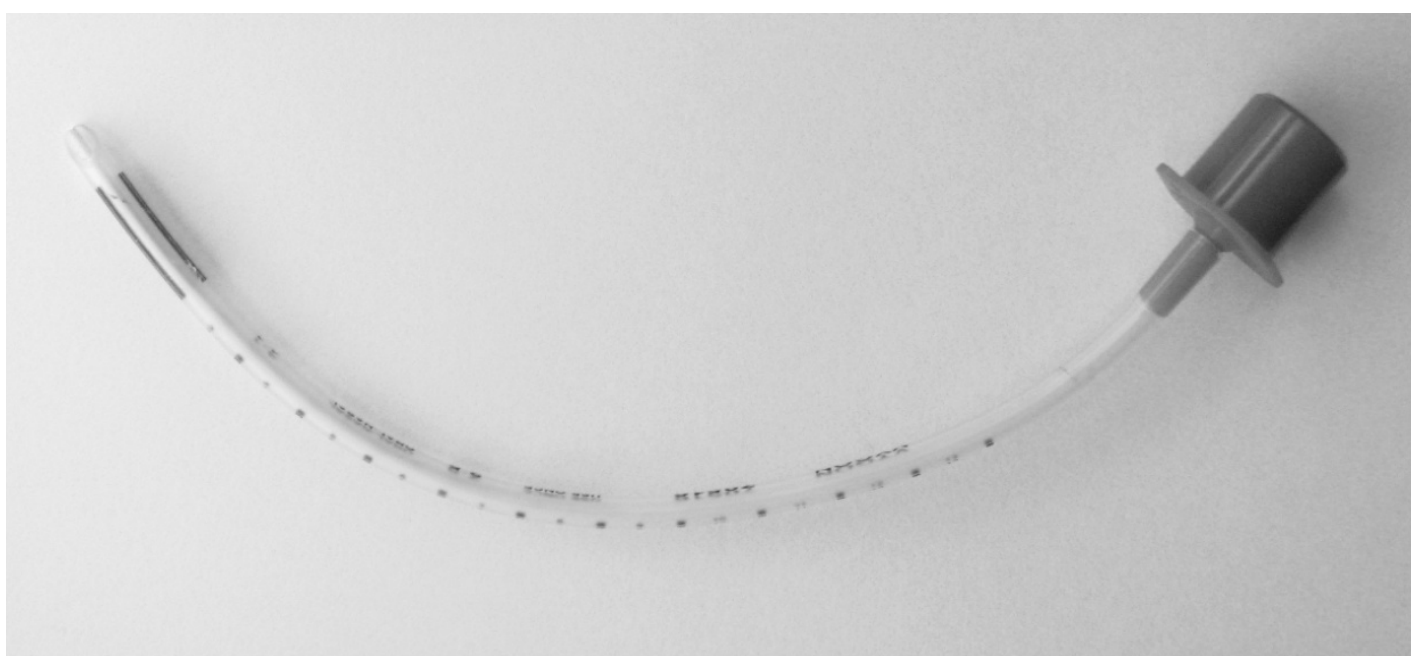

Fig. 1. Endotracheal tube

\subsection{Aerosol Particle Size Distribution Measurement}

The size distribution of aerosol droplets was determined by an on-line sampling of the aerosol leaving endotracheal tubes with the use of aerosol spectrometer GRIMM model 1109. The device counted droplets in 31 size channels of the $0.25-32 \mu \mathrm{m}$ size range utilising light scattering. The obtained number distribution was recalculated to cumulative mass distribution assuming known density and with the use of equation of the sphere volume. This allowed us to determine the fine particle fraction $(F P F)$ of aerosols leaving each of the endotracheal tubes. It was calculated by the interpolation of data showing the cumulative mass vs. droplet diameter (Fig. 2).

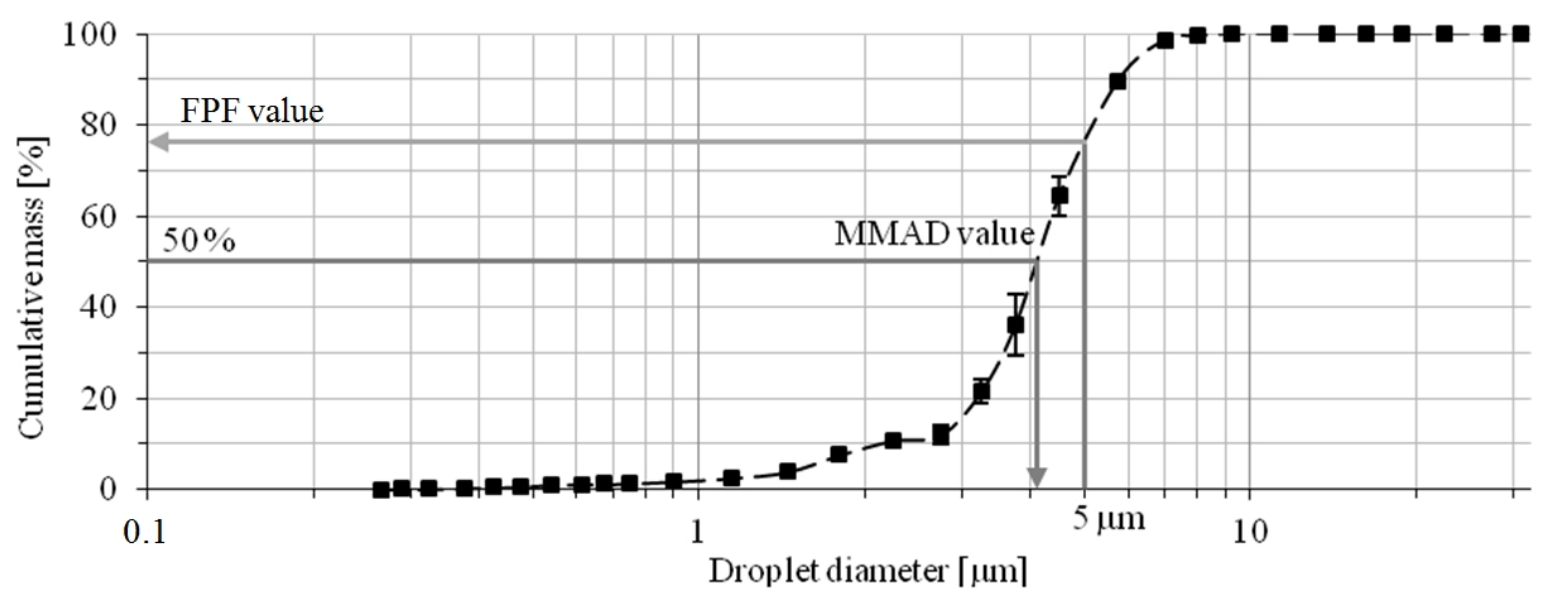

Fig. 2. Characterisation of the aerosol generated from nebuliser

$F P F$ is the fraction of droplets with aerodynamic diameter which is smaller than $5.0 \mu \mathrm{m}$. Droplets of this size range penetrate to the lower respiratory tract with the highest efficiency (Hickey, 1993; Gradon et al., 2007; Zanen et al., 1995). The mass median aerodynamic diameter (MMAD) of the examined droplets was defined on the basis of the same graph as the size of particles at which the line crosses the $50 \%$ mark. The geometric standard deviation $(G S D)$ was calculated as:

$$
G S D=\sqrt{\frac{X}{Y}}
$$

where $X$ is the diameter of droplets the mass of which is $84 \%$ of the total mass of droplets and where the $Y$ is the diameter of droplets the mass of which is $16 \%$ of their total mass. 


\subsection{Determination of DSCG mass delivered through ET tubes}

$7 \mathrm{ml}$ of $2.5 \%$ water solution of DSCG was used to fill up the nebuliser for each measurement. The amount of DSCG remaining in the nebuliser after measurement was determined spectrophotometrically at $340 \mathrm{~nm}$ against water as a reference sample. DSCG delivered through endotracheal tubes was collected in the tank with the previously known amount of water and its mass was determined spectrophotometrically as well.

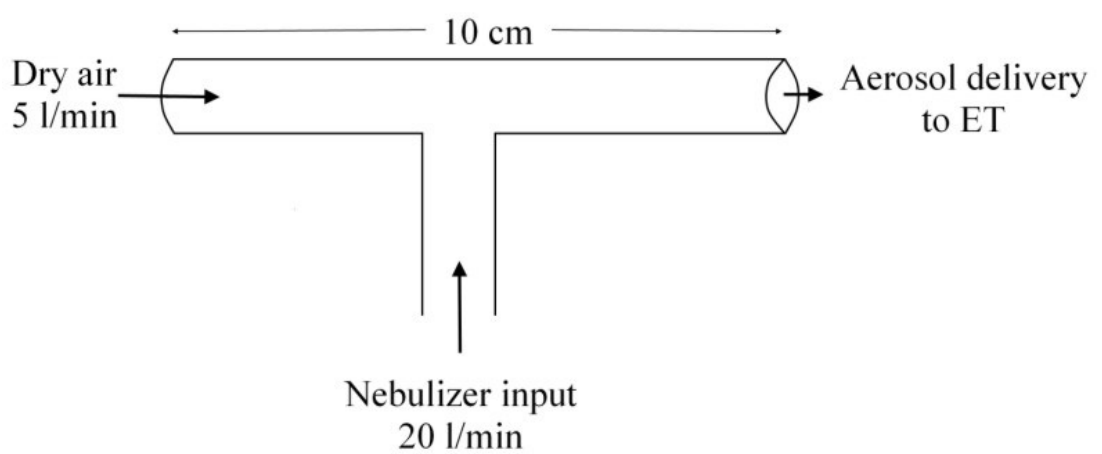

Fig. 3. Experimental setup of the system

All experiments were run in triplicate and their results were expressed as mean value \pm standard deviation.

\section{RESULTS AND DISCUSSION}

The mean value of medication mass delivered to the lower respiratory tract through endotracheal tubes is shown in Figure 4. Results were obtained after collection of drug deposited inside 16 endotrachel tubes. ET tubes had eight different inner diameters and were made from two types of materials (PVC and $\mathrm{PVC} /$ silicon). The medication dose penetrated into the lower respiratory tract in the range $19-33$ mg. In a simplified model of mechanical ventilation (constant airflow) $77 \pm 8 \%$ of inhaled dose penetrated through ET tubes made of PVC/silicon and $74 \pm 3 \%$ through ET tubes made of PVC. However, only about $15 \pm 2 \%$ of nominal dose placed in a nebuliser $(175 \mathrm{mg})$ penetrated through ET tubes, regardless of tube inner diameter or material (Fig. 5).

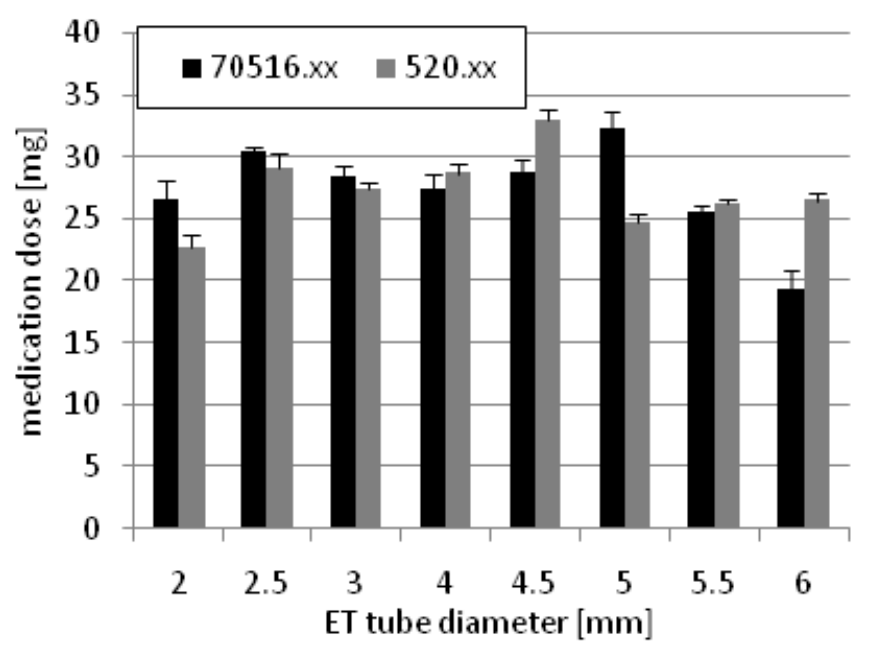

Fig. 4. The medication dose penetrated through ET tubes made of PVC (520.xx) or PVC/silicon (70516.xx).

Error bars represent the standard deviation from the three tests 


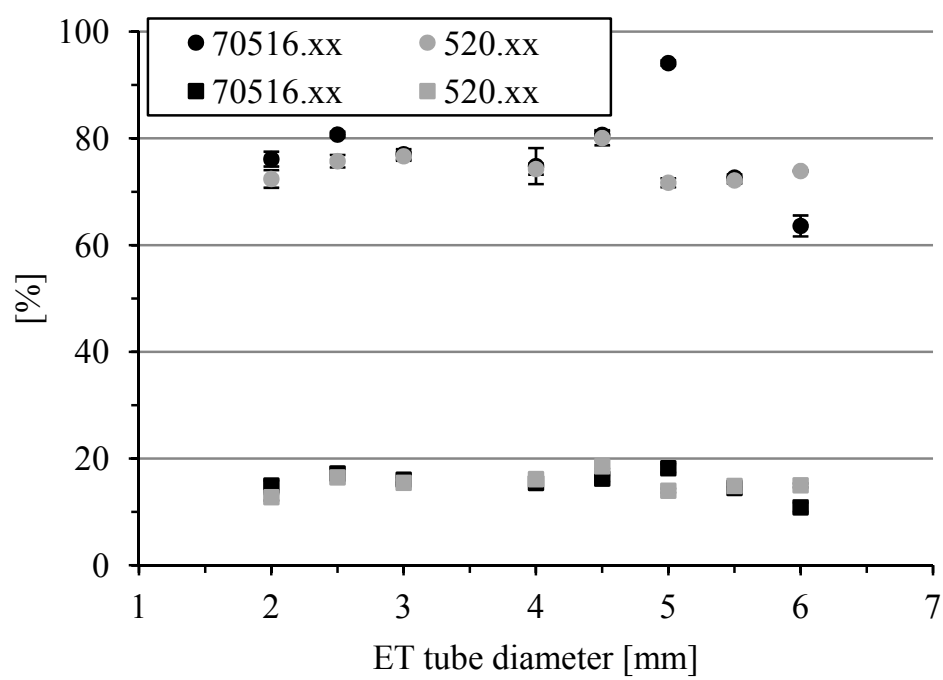

Fig. 5. Dots represent percentage of inhaled dose penetrated through ET tubes made of PVC (520.xx) or $\mathrm{PVC} /$ silicon (70516.xx), squares represent percentage of nominal dose placed in a nebuliser penetrated through ET tubes made of PVC (520.xx) or PVC/silicon (70516.xx). Symbols represent the mean values and the error bars represent the standard deviation from the three tests

Neither endotracheal tubes inner diameter nor the ET tubes material significantly influences the amount of medication delivered to intubated patient. In both cases, similar medication dose penetrated through ET tubes. The dose of medication delivered into the respiratory system (77 and $74 \%)$ is higher than the dose of the medicine delivered into the lungs in in vivo tests (Schueepp et al., 2009; Watterberg et al., 1991). Most of the in vivo tests are conducted at newborn infants where small endotracheal tubes are used and air flow rates are lower than $25 \mathrm{l} / \mathrm{min}$. Moreover, in our studies, constant flow was used whereas in mechanical ventilation respiratory curve is used (Cook et al., 1957; Grigg et. al., 1992). During expiration, a part of inhaled drug dose is removed from the respiratory tract This is why effective medication dose is lower than that in in vitro test.

It might be expected that high flow resistance (the smaller tube diameter the higher flow resistance) and higher gas linear velocity in endotracheal tubes with a small diameter influence aerosol deposition in ET tubes. However, no significant differences in medication dose penetrated through ET tubes with various diameter size and identical length $(2,2.5,3 \mathrm{~mm}$ and $4,4.5,5 \mathrm{~mm}$ and 5.5, $6 \mathrm{~mm}$ ) were observed.

The amount of DSCG penetrated through ET tubes with 5.5 and $6 \mathrm{~mm}$ diameter is lower than that in any other endotracheal tube. The explanation of this effect can be found when Mass Median Aerodynamic Diameter $(M M A D)$ and Fine Particle Fraction $(F P F)$ of aerosol at the end of endotracheal tube are compared. The obtained values of $F P F, M M A D$ and GSD are shown in Table 2.

$M M A D$ is decreasing and $F P F$ is increasing while endotracheal tube internal diameter increases (Figs. 6 and 7).

Many research studies demonstrate that aerosol droplets with a diameter of less than $5 \mu \mathrm{m}$ deposit in the lower respiratory tract (Gradoń et al., 2007; Heyder, 2004; Hickey, 1993; Zanen et al., 1995). However, as has been shown in our studies, there is only a low content of fine particles $(5-22 \%)$ and high $M M A D$ values $(15.9-21.7 \mu \mathrm{m})$ in aerosol leaving endotracheal tubes with the internal diameter of $2,2.5,3 \mathrm{~mm}$ and the length of $165 \mathrm{~mm}$. This suggests that effectiveness of treatment will be insufficient in this case. Endotracheal tubes with this range of internal diameter are commonly used in the treatment of neonates and infants. Additionally, neonates insufficiently developed lungs causing 
additional problems with the effectiveness of inhalation treatment. Probably the effectiveness of treatment may be improved by an appropriate adjustment of the air flow (air flow $3-81 / \mathrm{min}$ ).

Table 2. Fine Particle Fraction, \%, - FPF, Mass Median Aerodynamic Diameter, $\mu \mathrm{m}$, - MMAD, Geometric Standard Deviation - GSD, Standard Deviation - $S D$ for ET tubes made of PVC (520.xx) or $\mathrm{PVC} /$ silicon (70516.xx), average $\pm S D$, number of replicates $=3$

\begin{tabular}{|c|c|c|c|c|c|c|c|}
\hline $\begin{array}{c}\text { ET tube } \\
\text { diameter [mm] }\end{array}$ & Tube & $\begin{array}{c}F P F \\
{[\%]}\end{array}$ & $S D_{F P F}$ & $\begin{array}{c}M M A D \\
{[\mu \mathrm{m}]}\end{array}$ & $S D_{M M A D}$ & $\begin{array}{c}G S D \\
{[-]}\end{array}$ & $S D_{G S D}$ \\
\hline \multirow{3}{*}{2.0} & 70516.20 & 5.33 & 0.58 & 18.53 & 2.61 & 1.85 & 0.06 \\
\cline { 2 - 8 } & 520.20 & 7.47 & 0.45 & 15.90 & 0.17 & 1.56 & 0.01 \\
\hline \multirow{3}{*}{2.5} & 70516.25 & 8.03 & 0.40 & 21.67 & 0.76 & 1.77 & 0.03 \\
\cline { 2 - 8 } & 520.25 & 10.10 & 0.53 & 19.60 & 0.36 & 1.59 & 0.05 \\
\hline \multirow{3}{*}{3.0} & 70516.30 & 21.33 & 0.76 & 19.77 & 0.25 & 4.14 & 0.26 \\
\cline { 2 - 8 } & 520.30 & 22.83 & 1.76 & 19.33 & 0.61 & 4.18 & 0.05 \\
\hline \multirow{3}{*}{4.0} & 70516.40 & 36.33 & 1.53 & 10.17 & 0.29 & 4.94 & 0.32 \\
\cline { 2 - 8 } & 520.40 & 28.17 & 0.76 & 19.37 & 0.51 & 2.87 & 0.08 \\
\hline \multirow{3}{*}{4.5} & 70516.45 & 36.17 & 1.44 & 12.90 & 0.78 & 3.65 & 0.13 \\
\cline { 2 - 8 } & 520.45 & 46.50 & 3.12 & 9.83 & 2.75 & 4.16 & 0.22 \\
\hline \multirow{3}{*}{5.0} & 70516.50 & 39.17 & 1.04 & 12.17 & 0.29 & 7.25 & 0.51 \\
\cline { 2 - 8 } & 520.50 & 40.33 & 1.53 & 13.33 & 0.58 & 3.48 & 0.11 \\
\hline \multirow{3}{*}{5.5} & 70516.55 & 69.67 & 4.04 & 2.00 & 0.10 & 4.52 & 0.27 \\
\cline { 2 - 8 } & 520.55 & 72.67 & 3.51 & 3.57 & 0.12 & 3.68 & 0.09 \\
\hline \multirow{2}{*}{6.0} & 70516.60 & 90.17 & 0.76 & 3.17 & 0.15 & 1.68 & 0.06 \\
\cline { 2 - 8 } & 520.60 & 90.33 & 3.21 & 3.57 & 0.06 & 1.60 & 0.03 \\
\hline
\end{tabular}

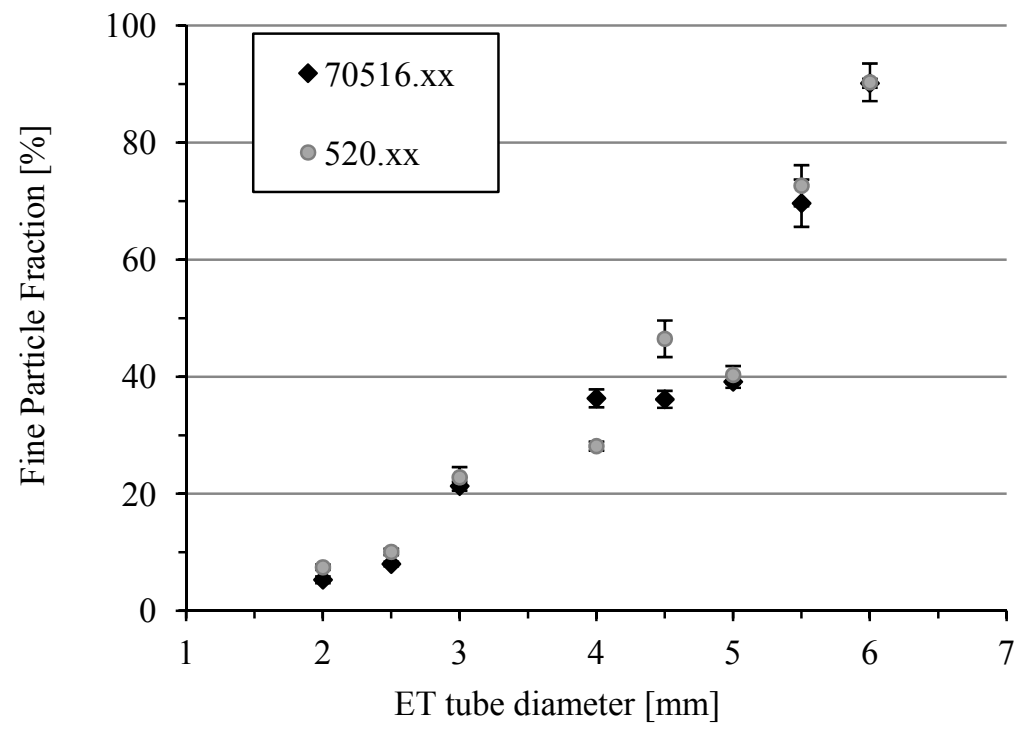

Fig. 6. Fine particle fraction (FPF, \%) of aerosol delivered through ET tubes made of PVC (520.xx) or PVC/silicon (70516.xx) 


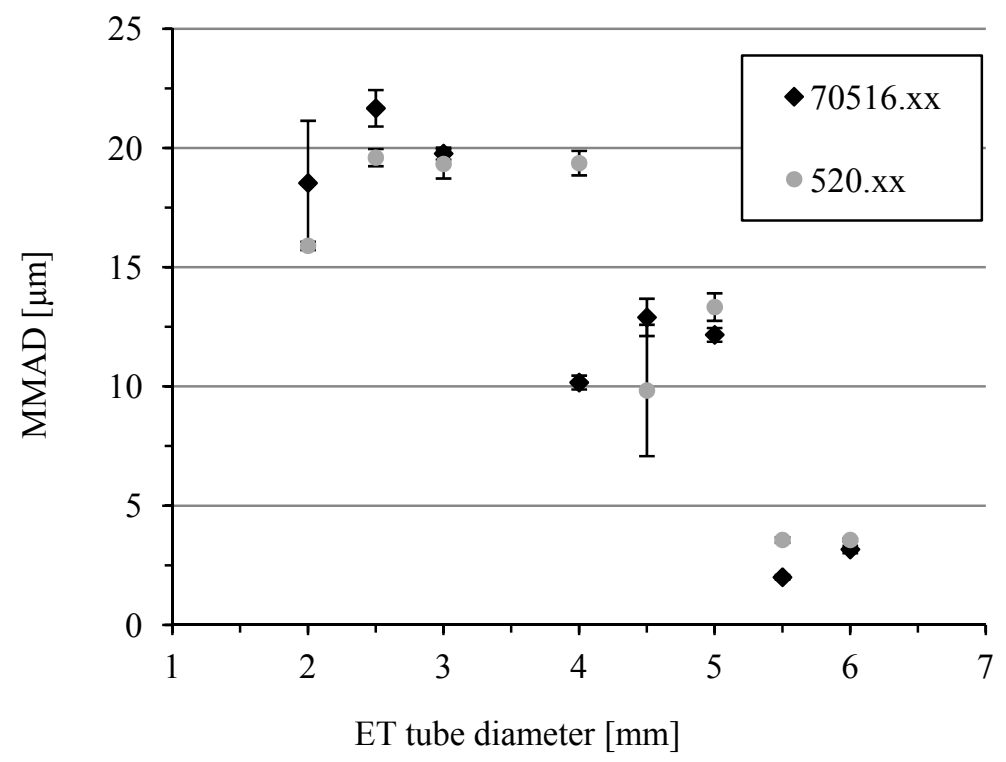

Fig. 7. Mass median aerodynamic diameter $(M M A D, \mu \mathrm{m})$ of aerosol delivered through ET tubes made of PVC (520.xx) or PVC/silicon (70516.xx)

Aerosols leaving endotracheal tubes with a large internal diameter $(5.5,6 \mathrm{~mm})$ and the highest length $(270 \mathrm{~mm})$ have low $M M A D$ values $(2-3.5 \mu \mathrm{m})$ and high values of $F P F(69-90 \%)$. These parameters are therefore equal or even better than those for aerosol emitted directly from a nebuliser $(M M A D=4.1 \mu \mathrm{m}$ and $F P F=74 \%)$ and it is probably related to a deposition of large droplets on the surface of tubes. A decrease of the mass of DSCG deposited in the tank confirms this hypothesis. This effect is particularly noticeable for long endotracheal tubes. Aerosol leaving ET tubes with an internal diameter of $5 \mathrm{~mm}$ and $210 \mathrm{~mm}$ length has the $M M A D$ value that is equal to $13 \mu \mathrm{m}$ and $F P F-40 \%$. An extension of tube`s length to $270 \mathrm{~mm}$ causes an improvement of aerosol parameters $(M M A D=3 \mu \mathrm{m}$ and $F P F=70-90 \%)$.

A comparison of values of the MMAD and FPF (Table 2) as well as the mass of DSCG deposited in the tank (Fig. 4) for different tube materials (PVC or PVC/silicon) shows no significant influence of the type of the material. Still, their elasticity or tendency to cause allergies may become a criterion of their choice, but this was not the goal of our studies.

\section{CONCLUSIONS}

The material that the tubes are made of has a remote influence on aerosol delivery during mechanical ventilation of adults. The amount of medication penetrated through endotracheal tubes of a different diameter is almost equal. However, the effective drug dose delivered into the respiratory tract is lower if small endotracheal tubes are used. $M M A D$ and $F P F$ for large ET tubes were almost identical with $M M A D$ and $F P F$ from nebuliser. In this case, the effectiveness of pulmonary drug delivery did not vary.

Aerosol delivery of drugs to intubated patients has not been well examined yet. Most researchers focus on ventilation of newborn infants or small babies. To the best of our knowledge, our study is one of few attempts to examine the issue of delivering medication to intubated adults. 


\section{REFERENCES}

Ahrens R.C, Ries R.A, Popendorf W, Wiese J.A., 1986. The delivery of therapeutic aerosols through endotracheal tubes. Pediatr. Pulmonol., 2, 19-26. DOI: DOI: 10.1002/ppul.1950020108.

Cook C.D., Sutherland J.M., Segal S., Cherry R.B., Mead J., McIlroy M.B., Smith C.A., 1957. Studies of respiratory physiology in the newborn infant. III Measurements of mechanics of respirations. J. Clin. Invest., 36, 440-448. DOI: $10.1172 / \mathrm{JCI} 103441$.

Dhand R., 2000. Special problems in aerosol delivery: Artificial airways. Respir. Care, 45, 636-645.

Gradoń L., Moskal A., Sosnowski T.R., 2007. Estimation of separation efficiencies of aerosol particles in the upper parts of a human respiratory tract. Chem. Process Eng., 28, 465-473.

Grigg J., Arnon S., Jones R., Clarke A., Silverman M., 1992. Delivery of therapeutic aerosols to intubated babies. Arch. Dis. Child., 67, 25-30. DOI: 10.1136/adc.67.1_Spec_No.25.

Heyder J., 2004. Deposition of inhaled particles in the human respiratory tract and consequences for regional targeting in respiratory drug delivery. Proc. Am. Thorac. Soc., 1, 315-320. DOI: 10.1513/pats.200409-046TA.

Hickey A.J., 1993. Lung deposition and clearance of pharmaceutical aerosols: What can be learned from inhalation toxicology and industrial hygiene? Aerosol Sci. Technol., 18, 290-304. DOI: $10.1080 / 02786829308959606$.

MacIntyre N.R, Silver R.M, Miller C.W, Schuler F., Coleman R.E., 1985. Aerosol delivery in intubated, mechanically ventilated patients. Crit. Care Med., 13, 81-84.

Mazela J., Sosnowski T.R., Moskal A., Gadzinowski J., 2011. Small neonatal ET Tube sizes decrease aerosol penetration - Computational Fluid Dynamics study. Respiratory Drug Delivery Europe 2011, Berlin, Germany, 2-6 May 2011, 2, 401-404.

Schueepp K.G., Devadason S.G., Roller Ch., Minoccheieri S., Moeller A., Hamacher J., Wildhaber J. H., 2009. Aerosol delivery of nebulised budesonide in young children with asthma. Respir. Med., 103, 1738-1745. DOI: 10.1016/j.rmed.2009.04.029.

Sosnowski T.R., 2010. Inhalable aerosols and inhalers. WIChiP PW, Warsaw University of Technology, 14 (in Polish).

Watterberg K., Clark A., Kelly H.W., Murphy S, 1991. Delivery of aerosolized medication to intubated babies. Pediatr. Pulmonol., 10, 136-141. DOI: 10.1002/ppul.1950100217.

Zanen P., Go L.T., Lammers J.J., 1995. The optima particie size for parasympathicolytic aerosols In mild asthmatics, Int. J. Pharmaceutics, 114, 111-115. DOI: 10.1016/0378-5173(94)00224-S.

Żywczyk Ł., Moskal A., Sosnowski T.R., 2010. Badanie depozycji cząstek aerozolowych w rurkach dozujących (Endotracheal tube) stosowanych podczas podawania leków. Inż. Aparat. Chem., 49, 125-126. 